\title{
Assessment Of Tumour Necrosis Factor-Alpha (Tnf- A) And Creatinine Levels In Echis Ocellatus Bite Victims In Jos Metropolis, Nigeria
}

\author{
Manafa P.O. \\ Osmond E.O. \\ Onyenekwe C.C. \\ Okeke C.O. \\ Chukwuma G.O. \\ Ihim A.C. \\ Ogenyi S.I. \\ Chukwuanukwu R.C. \\ Department of Medical of Medical Laboratory Science, Faculty of Health \\ Sciences and Technology, Nnamdi Azikiwe University, Nnewi Campus, \\ Anambra State. \\ Manafa C.C. \\ Ministry of Health, Rivers state, Nigeria. \\ Nnadi E.C \\ Department of Medical of Medical Laboratory Science, Faculty of Health \\ Sciences and Technology, Nnamdi Azikiwe University, Nnewi Campus, \\ Anambra State. \\ doi: 10.19044/esj.2016.v12n21p70 URL:http://dx.doi.org/10.19044/esj.2016.v12n21p70

\begin{abstract}
This study was designed to assess tumour necrosis factor-alpha and creatinine levels in Echis ocellatus bite victims. A total of 50 subjects were recruited. Out of this number, 40 were victims of E. ocellatus bite and the remaining 10 were non-victims of snake bite who served as the control group. Blood samples were collected from the victims within 24 hours of the snake bite and EchiTAb-G antivenom administered within the same period. Another batch of blood sample was collected 48 hours post-administration of the anti-venom. Tumour necrosis factor-alpha (TNF-alpha) levels were estimated by the Enzyme Linked Immunosorbent Assay technique while creatinine levels were determined using kinetic-spectrophotometric procedure. The mean serum levels of tumour necrosis factor-alpha and creatinine were significantly increased in E. ocellatus bite victims compared
\end{abstract}


with the control group $(\mathrm{P}<0.05)$. Furthermore, the mean serum level of TNFalpha was significantly lower in E. ocellatus bite victims, post-administration of anti-venom, compared with the pre-administration of anti-venom $(\mathrm{P}<0.05)$. In contrast, no significant difference was observed in the mean serum level of creatinine in E. ocellatus bite victims, post-administration of anti-venom, compared with the pre-administration of anti-venom $(\mathrm{P}>0.05)$. Moreover, the mean serum level of creatinine was found to be significantly increased in E. ocellatus bite victims, post-administration of anti-venom, compared with the control group $(\mathrm{P}<0.05)$, while no significant difference was observed in the mean serum level of tumour necrosis factor-alpha in $E$. ocellatus bite victims, post-administration of anti-venom, compared with the control group $(\mathrm{P}>0.05)$. A positive correlation existed between tumour necrosis factor-alpha and creatinine levels in E. ocellatus bite subjects ( $\mathrm{r}=$ 0.782). Echis ocellatus bite is a risk factor for renal damage indicated by an elevated serum creatinine, thus health authorities should make EchiTAb-G anti-venom freely available in health facilities and administered as quickly as possible to reduce the risk of renal damage in Echis ocellatus bite-prone areas.

Keywords: Tumor necrosis factor alpha, creatinine, Snake venoms, antivenoms, Echis ocellatus

\section{Introduction}

Snakebite envenoming comprises a major public health problem among communities of the savanna region of West Africa, notably in Benin, Burkina-Faso, Cameroon, Ghana, Nigeria and Togo (Chippaux et al., 2007). Four families of venomous snakes are found in Nigeria--Viperidae, Elapidae, Colubridae and Actraspididae but three species carpet viper (Echis ocellatus), black-necked spitting cobra (Naja nigricollis) and puff adder (Bitis arietans), belonging to the first two families, are the most important snakes associated with envenoming in Nigeria, with the saw-scaled or carpet viper (Echis ocellatus) as the most important cause of snakebite mortality and morbidity in the region (Habib et al., 2001). Envenomation by snakes of the family Viperidae is characterized by prominent local effects, including necrosis, hemorrhage, edema, and pain, which develops rapidly after the accident and often result in permanent sequelae (Warrell, 1995) and systemic alterations such as hemorrhage, coagulopathy, shock, and acute renal failure, may occur. Both local and systemic effects of these snake venoms have been associated with the action of a variety of venom components, notably metalloproteinases (Gutierrez and Lomonte, 1997).

Tumor necrosis factor- alpha (TNF- $\alpha$ ) is a proinflammatory cytokine produced mainly by activated macrophages or monocytes and plays an 
important role in diverse cellular events, such as the production of other cytokines, cell proliferation, differentiation and apoptosis (Beyaert and Fiers, 1994). The cellular changes in response to TNF- $\alpha$ are cell-type dependent. For example, TNF- $\alpha$ may modify the anticoagulant properties of endothelial cells, promote $\mathrm{T}$ cell proliferation, cause bone resorption, and induce the release of other inflammatory cytokines in many different cells.

TNF- $\alpha$ is not usually detectable in healthy individuals, however, elevated serum and tissue levels are found in inflammatory and infectious conditions (Robak et al., 1998) and serum levels correlate with the severity of infections. Inappropriate production of TNF- $\alpha$ has been implicated in the pathogenesis of both acute and chronic inflammatory diseases such as septic shock, AIDS, arthritis and cancer (Tracey and Cerami, 1993).

Creatinine (MW 113 D) is the cyclic anhydride of creatine that is produced as the final product of decomposition of phosphocreatine. Creatine is synthesized in the kidneys, liver, and pancreas, primarily in the liver from arginine, glycine, and methionine and then transported to other tissues, such as muscle, where it is converted to phosphocreatine, which serve as highenergy source. Creatine phosphate loses phosphoric acid and creatine loses water to form creatinine, which passes into the plasma. Plasma creatinine concentration is a function of relative muscle mass and renal function (Bishop et al., 2005). It remains within the reference interval until significant renal function has been lost.

This study was therefore designed to assess tumor necrotic factor alpha and renal damage in Echis ocellatus bite victims.

\section{Materials and methods}

Study sites

This research was carried out at Nnamdi Azikiwe University Teaching Hospital, Nnewi, Anambra state, Nigeria and Jos University Teaching Hospital Comprehensive Health Centre Zamko, Plateau State, Nigeria.

\section{Study population}

A total of 50 individuals were recruited for this study which included 40 Echis ocellatus bite subjects and 10 non-victims of snake bite which served as the control group. Samples were collected prior to anti-venom administration and 48 hours post-administration of the anti-venom from the victims of Echis ocellatus bite.

\section{Exclusion criteria}

Patients with renal disorders, inflammatory and autoimmune diseases such as Systemic lupus erythematosus (SLE), Scleroderma, Sjögren's 
syndrome, Mixed connective tissue disease, Polymyositis/dermatomyositis and Rheumatoid arthritis were excluded in the study.

\section{Ethical consideration and informed consent}

The ethical approval for this research was obtained from the ethics committee of the Faculty of Health Science and Technology, College of Health Sciences, Nnamdi Azikiwe University, Nnewi campus and informed consent of the subjects were obtained.

\section{Collection of samples}

About 5ml of venous blood was collected aseptically from each subject and dispensed into a plain container for the determination of tumour necrosis factor-alpha and creatinine levels. The samples were centrifuged at 5,000 rpm for 5 minutes and the serum separated.

\section{Analysis of parameters}

The method employed in the estimation of tumour necrosis factor alpha level was an enzyme immunosorbent assay as described by Meade et al (1986) while plasma creatinine level was estimated using kinetic Jaffe reaction as described by Lipitskaia et al. (1989).

\section{Statistical analysis}

Statistical package for Social sciences (SPSS) version 20 was used for analysis using the students t-test and Pearson correlation. Values were deemed significant if $\mathrm{p}<0.05$

\section{Results}

The mean serum level of tumour necrosis factor-alpha in Echis ocellatus bite victims, pre-administration of anti-venom was significantly increased compared with the control subjects $(p<0.05)$. Similarly, a significant increase was observed in the mean serum level of creatinine in Echis ocellatus bite victims, pre-administration of antivenom, compared with the control subjects $(\mathrm{p}<0.05)$ (Table 1$)$.

There was a significant decrease in the mean serum level of tumour necrosis factor-alpha (TNF- $\alpha$ ) post-administration of anti-venom, compared with the pre-administration of anti-venom, in the Test subjects $(p<0.05)$. Statistically no significant difference existed in the mean serum level of creatinine post-administration of anti-venom, compared with preadministration of anti-venom, in the Test group ( $\mathrm{P}>0.05)$ (Table 2).

There existed no significant difference in the mean serum level of tumour necrosis factor-alpha in Echis ocellatus bite victims, postadministration of anti-venom, compared with the control subjects $(P>0.05)$. 
However, a significant increase was observed in the mean serum level of creatinine in Test group, post-administration of antivenom, compared with the control subjects $(\mathrm{p}<0.05)$. (Table 3$)$

In figure 1 , a positive correlation was observed between tumour necrosis factor-alpha and creatinine levels in Echis ocellatus bite victims preadministration of antivenom ( $\mathrm{r}=0.782)$.

Table 1: Comparison of tumour necrosis factor-alpha (TNF- $\alpha$ ) and creatinine levels in control group and Echis ocellatus bite victims (Test group) pre-administration of anti-

venom.

\begin{tabular}{cccc}
\hline GROUPS & $\mathrm{N}$ & Tumour Necrosis factor $(\mathrm{ng} / \mathrm{ml})$ & Creatinine $(\mu \mathrm{mol} / \mathrm{l})$ \\
\hline $\begin{array}{c}\text { Echis ocellatus bite } \\
\text { victims }\end{array}$ & 40 & $42.87 \pm 42.22$ & $106.39 \pm 12.25$ \\
Control subjects & 10 & $6.39 \pm 10.12$ & $85.06 \pm 17.67$ \\
P-value & & $0.02^{*}$ & $0.01 *$ \\
\hline
\end{tabular}

Table 2: Comparison of tumour necrosis factor-alpha and creatinine levels in pre and postadministration of anti-venom in the Test group.

\begin{tabular}{cccc}
\hline GROUP & $\mathrm{N}$ & Tumour Necrosis factor $(\mathrm{ng} / \mathrm{ml})$ & Creatinine $(\mu \mathrm{mol} / \mathrm{l})$ \\
\hline Pre-treatment & 40 & $42.87 \pm 42.22$ & $106.39 \pm 12.25$ \\
Post-treatment & 10 & $18.68 \pm 31.38$ & $101.49 \pm 17.93$ \\
P-value & & $0.02^{*}$ & 0.16 \\
\hline
\end{tabular}

Table 3: Comparison of tumour necrosis factor-alpha (TNF- $\alpha$ ) and creatinine levels in control subjects and Echis ocellatus bite victims post-administration of anti-venom.

\begin{tabular}{cccc}
\hline GROUP & $\mathrm{N}$ & Tumour Necrosis factor $(\mathrm{ng} / \mathrm{ml})$ & Creatinine $(\mu \mathrm{mol} / \mathrm{l})$ \\
\hline Post-treatment & 40 & $18.68 \pm 31.38$ & $101.49 \pm 17.93$ \\
Control & 10 & $6.39 \pm 10.12$ & $85.06 \pm 17.67$ \\
P-value & & 0.17 & $0.04^{*}$ \\
\hline
\end{tabular}

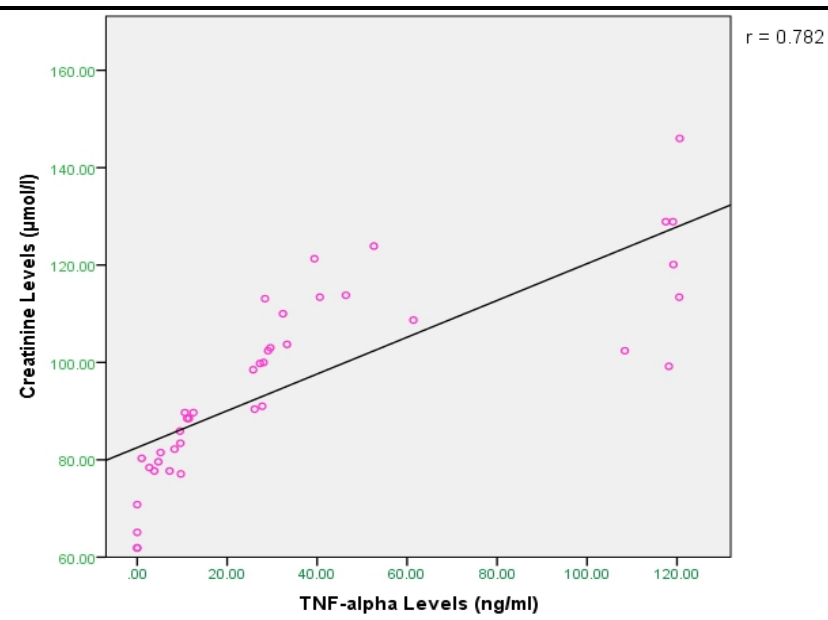

Fig 1: Correlation of tumour necrosis factor-alpha and creatinine levels in Echis ocellatus bite victims pre-administration of antivenom. 


\section{Discussion}

Snakebite envenomation is a neglected tropical disease that affects each year, hundreds of thousands of individuals in tropical and sub-tropical areas of the world (Kasturiratne et al., 2008). In the north-eastern and central parts of Nigeria, Carpet viper (Echis ocellatus) along with other true and pit vipers, is the venomous snake responsible for most deaths in snakebite cases. Carpet viper venom induces a pathophysiological picture, characterized by immediate and prominent local tissue damage (myonecrosis, edema, inflammation, haemorrhage and hypovolemic shock) and coagulation disorders (defibrination) which might end in acute renal failure (Amoral et al., 1985).

The parenteral administration of animal-derived anti-venoms constitutes the mainstay in the therapy of snakebite envenoming (WHO, 2010). In Nigeria, EchiTAb-G (a monospecific antivenom manufactured by Micropharm) developed against the venom of the Nigerian snake (Echis ocellatus) is used in the management of envenoming by this specie (Abubakar et al., 2010). Restoration of blood coagulability has been used as a surrogate marker of antivenom effectiveness in many clinical studies of viper-bite-induced consumption coagulopathy (Viser et al., 2008). In this study, we assessed the capacity of Echis ocellatus venom to cause inflammation and/or renal damage and the efficacy of ECHITab-G in neutralizing these inflammatory and nephrotoxic effect of the snake venom. The mean serum level of tumour necrosis factor-alpha was significantly increased in $E$. ocellatus bite victims pre-administration of anti-venom, compared with the control group $(\mathrm{P}<0.05)$. Similarly, a significant increase was observed in the mean serum level of creatinine in E. ocellatus bite victims pre-administration of anti-venom, when compared with that of the control ( $\mathrm{P}<0.05)$. Crocker et al (2010) and Moreira et al (2012), had earlier reported elevated plasma concentrations of TNF- $\alpha$ and other proinflammatory cytokines such as IL-6, IL-8 in envenomed humans. TNF is produced predominantly by activated macrophages and $\mathrm{T}$ lymphocytes as a $26 \mathrm{kDa}$ protein, pro-TNF, which is expressed on the plasma membrane, where it can be cleaved in the extracellular domain by the matrix metalloproteinases, resulting in the release a soluble active $17 \mathrm{kDA}$ form (Wang et al., 2003). Moura-da-Silva et al(1996) have shown that two venom zinc metalloproteinases (jararhagin from Bothrops jararaca venom and a metalloproteinase from Echis pyramidum leakeyi venom) successfully cleaved the recombinant glutathione-S-transferase-tumor necrosis factoralpha fusion protein (GST-TNF-alpha) substrate to form biologically active TNF-alpha. This cytokine may contribute to the local necrosis and also induce the production of endogenous matrix metalloproteinases, which in turn generate a positive feedback mechanism resulting in continued cleavage 
of pro-TNF-alpha. These circumstances may be the cause of the elevated plasma concentration of TNF-alpha.

The elevations in creatinine level in the test subjects, preadministration of antivenom, observed in the present study are similar to that reported by Kale and Lonkar (2013), where the creatinine level was found to be 2.6 times the level of the control subjects. Muhammad (2009) also demonstrated significantly increased levels of serum creatinine in Guinea pigs envenomated with Echis coloratus and noted that this crude venom caused hepatic and renal dysfunction in the Guinea pigs. Furthermore, Gutie'rrez and Ownby (2003) and Montecucco et al (2008) reported that the venom of vipers contain myotoxic PLA2s and PLA2 homologues which induce rapid alterations to the plasma membrane of the muscle cells (myotoxicity), followed by irreversible cell injury. The myotoxic activity of the venom produces severe skeletal and cardiac muscle injury leading to myalgia and rhabdomyolysis with the subsequent release of myoglobin from damaged skeletal muscle into serum leading to myoglobinemia with further myoglobinuria which can result in damage to the kidneys (nephrotoxicity) as a result of myoglobin accumulation in the renal tubules. This is coupled with hypotension and may lead to acute renal failure - the cause of death among patients surviving to the early effects of snakebites (Markus et al., 2012). Zhou et al (2008) also included direct nephrotoxicity of the snake venom, circulatory collapse, intravascular hemolysis and microangiopathic hemolytic anaemia as other contributing factors that could lead to acute renal disease observed in snake envenomation.

Furthermore, this study observed a significantly decreased mean serum level of tumour necrosis factor-alpha in Echis ocellatus bite victims, post-administration of antivenom, compared with the pre-administration of antivenom. However, there was no significant difference in the mean serum level of creatinine in Echis ocellatus bite victims, post-administration of antivenom compared with the pre-administration of antivenom ( $\mathrm{P}>0.05)$.

Juan et al (2010), while assessing the immunological reactivity of EchiTAb-Plus-ICP, revealed a complete immunodepletion of the majority of venom components, including metalloproteinases, serine proteinases, C-type lectin-like proteins, some phospholipases A2 and L-amino acid oxidase.

In contrast, Stone et al (2013) found that after treatment with antivenom in Sri Lankan viper envenoming there were further increases in TNF- $\alpha$ and other cytokines(IL-6, IL-10), though, typical hypersensitivity reactions to antivenom occurred in $64 \%$ of the patients which correlated with severity of hypersensitivity reactions and strongly suggested that the increase reflects an immune response to the antivenom.

In this work, no significant difference was observed in the mean serum level of creatinine in the test group, post-administration of antivenom, 
compared with the pre-administration of antivenom. Thein-Than et al (1991) had earlier reported that renal damage can develop very early and even when the patient arrives at hospital soon after the bite, the damage may already have been done. They further observed that even when anti-snake venom was administered within 1-2 hours after the snake bite, it was incapable of preventing acute renal failure. To buttress this, Warrell (1999) maintained that a victim in renal failure is evidence of the previous action of venom either directly on the kidney or by fibrin deposition and does not necessarily imply that the victim currently has un-neutralised venom in the system. Hence, Shastry et al (1977) suggested that declining renal parameters in snake bite victims require referral to a specialist nephrologist where either peritoneal dialysis or haemodialysis could be performed.

Statistically, no significant difference was observed in the mean serum level of tumour necrosis factor-alpha in E. ocellatus bite victims, post administration of antivenom, compared with the control group while a significant increase was observed in the mean serum level of creatinine in $E$. ocellatus bite victim, post-administration of antivenom compared with the control group. This futher illustrates the capacity of the anti-venom in neutralizing the inflammatory effects of the snake venom and its inability to reverse renal damage already caused by the snake venom.

A positive correlation was observed between tumour necrosis factoralpha and creatinine levels in E. ocellatus bite victims, pre-administration of antivenom ( $\mathrm{r}=0.782)$. It has been recorded that in addition to the hemorrhagic effect of SVMP, they are also involved in the pathogenesis of myonecrosis that could lead to increased renal damage and inflammatory reactions releasing inflammatory cytokines like TNF-alpha (Moura-da-Silva et al., 1996). This implicates the action of snake venom metal as the major cause of this positive correlation observed between tumour necrosis factor-alpha and creatinine levels.

\section{Conclusion}

In this study, we have been able to show that tumour necrosis factoralpha and creatinine levels were significantly increased in Echis ocellatus evenomation. This implies that Echis ocellatus evenomation is associated with alterations in renal function markers and inflammatory mediators. Thus envenomed subjects are at higher risks of acute kidney impairment.

\section{Recommendations}

Baseline and post assessment of the inflammatory and renal status of Echis ocellatus bite victims is recommended. EchiTAb-G antivenom should be made freely available in Echis ocellatus bite-prone areas and administered as quickly as possible to reduce the risk of renal damage associated with 
Echis ocllatus evenomation.

\section{References:}

Abubakar, S.B., Abubakar, I.S., Habib, A.G., Nasidi, A., Durfa, N., Yusuf, P.O. (2010). Pre-clinical and preliminary dose-finding and safety studies to identify candidate antivenoms for treatment of envenoming by saw-scaled or carpet vipers (Echis ocellatus) in northern Nigeria. Toxicon. 55(4):719-723. Amoral, C. F., da Silva, O. A., Goody, P., Miranda, D. (1985). Renal cortical necrosis follwong Bothrops jararaca and B. jararacussu snakebite. Toxicon. 23:877-885.

Beyaert, R., Fiers, W. (1994). Molecular mechanisms of tumor necrosis factor induced cytotoxicity. What we do understand and what we do not. Federation of European Biochemical Societies.340: 9-16.

Bishop, M.L., Janet, L., Duben-Engelkirk, Fody, E.P. (2005). Clinical Chemistry, principles, procedures, correlations. (5th edition). London: Lippincott Williams and Wilkins. Pp 224-532.

Chippaux, J.P., Massougbodji, A., Stock, R.P., Alagon, A. (2007). Investigators of African antivipmyn in Benin: Clinical trial of an F(ab')2 polyvalent equine antivenom for African snake bites in Benin. American Journal of Tropical Medicine and Hygiene. 77(3): 538-546.

Crocker, P,, Zad, O., Milling, T., Maxson, T., King, B. (2010). Human cytokine response to Texas crotaline envenomation before and after antivenom administration. American Journal of Emergency Medicine. 28:871-879.

Gutiérrez, J.M., Lomonte, B. (1997). Phospholipase $A_{2}$ myotoxins from Bothrops snake venoms. In Kini, R.M. Venom Phospholipase $A_{2}$ Enzymes. Structure, Function and Mechanism.p.321-352.

Gutie'rrez, J.M., Ownby, C.L. (2003). Skeletal muscle degeneration induced by venom phospholipases A2: insights into the mechanisms of local and systemic myotoxicity. Toxicon. 42: 915-931.

Habib, A.G., Onyemelukwe, G.C., Gebi, U.I. (2001). Snake bite in Nigeria. African Journal of Medicine and Medical Science.30(3): 171-178.

Juan J. C., Pedro C., Libia S., Álvaro S., Mauren V., María H., Guillermo L., Robert H., Nandul D., Abdusalami N., Theakston R.G., David A. W., Gutiérrez, J.M. (2010). Antivenomic Assessment of the Immunological Reactivity of EchiTAb-Plus-ICP, an Antivenom for the Treatment of Snakebite Envenoming in Sub-Saharan Africa. American Journal of Tropical Medicine and Hygiene. 82(6):1194-1201.

Kale, B., Lonkar, A. (2013). Blood Hemoglobin, Lactate Dehydrogenase And Total Creatine Kinase Combinely As Markers Of Hemolysis And Rhabdomyolysis Associated With Snake Bite. International Journal of Toxicological and Pharmacological Research. 5(1): 5-8. 
Kasturiratne, A., Wickremasinghe, A.R., de Silva N., Gunawardena N.K., Pathmeswaran A. (2008). The global burden of snakebite: a literature analysis and modeling based on regional estimates of envenoming and deaths. PLoS Medicine.5:218.

Lipitskaia I.I, Kotkina T.i, Tarasov A.V, Titov V.N. (1989) A kinetic method of determining creatinine using jaffe reaction. Lab Delo. 2:37-42.

Markus, B., Maria, A. R. V., Jorge, A. G. (2012). Acute Kidney Injury Induced by Snake and Arthropod Venoms. In: Renal failure - the fact. $\left(2^{\text {nd }}\right.$ edition). Europe: Intech. Pp 157-186.

Meade, T.W., Mellows, S., Brozovic, M., Miller, G.J., Chakrabarti, R.R., North, W.R., Haines, A.P., Stirling, Y., Imeson, J.D., Thompson, S.G. (1986). Haemostatic function and ischaemic heart disease: principal results of the Northwick Park Heart Study. Lancet. 2: 533-537.

Montecucco, C., Gutie'rrez, J.M., Lomonte, B. (2008). Cellular pathology induced by snake venom phospholipase A2 myotoxins and neurotoxins: common aspects of their mechanisms of action. Cellular and Molecular Life Sciences. 65:2897-2912.

Moreira, V., Dos-Santos, M.C., Nascimento, N.G., Borges da Silva, H., Fernandes, C.M.(2012). Local inflammatory events induced by Bothrops atrox snake venom and the release of distinct classes of inflammatory mediators. Toxicon.60:12-20.

Moura-da-Silva, A.M., Laing, G.D., Paine, M.J., Dennison, J.M., Politi, V., Crampton, J.M., Theakston, R.D. (1996). Processing of pro-tumor necrosis factor-alpha by venom metalloproteinases: a hypothesis explaining local tissue damage following snake bite. European Journal of Immunology.26(9):2000-2005.

Muhammad, M. A. S. (2009). Physiological effects of envenomation by two different doses of the viper Echis coloratusis crude venom on biochemical parameters in serum of Guinea pigs atdifferent times. Egyptian Academic Journal of biological Sciences. 1(1): 21-31.

Robak, T., Gladalska, A., Stepien, H. (1998). The tumour necrosis factor family of receptors/ligands in the serum of patients with rheumatoid arthritis. European Cytokine Network. 9(2):145-154.

Shastry, J.C.M., Date, A., Carman, R.H., John, K.V. (1977). Renal failure following snake bite. American Journal of Tropical Medicine and Hygiene. 26:1032-1038.

Stone, S.F., Isbister, G.K., Shahmy, S., Mohamed, F., Abeysinghe, C. (2013). Immune Response to Snake Envenoming and Treatment with Antivenom; Complement Activation, Cytokine Production and Mast Cell Degranulation. PLoS Neglected Tropical Diseases.7(7): 2326.

Thein-Than, Tin-Tun, Hla-Pe, Phillips, R.E, Myint-Lwin, Tin-Nu-Swe, Warrell, D.A. (1991). Development of renal function abnormalities following 
bites by Russell's vipers (Daboia russelii siamensis) in Myanmar. Transactions of the Royal Society ofTropical Medicine and Hygiene. 85(3):404-409.

Tracey, K.J., Cerami, A. (1993). Tumor necrosis factor,other cytokines and disease. Annual Review Cell Biology. 9: 317-343.

Visser, L.E., Kyed-Faried, S., Belcher, D.W., Geelhoed, D.W., van Leeuwen, J.S., van Roosmalen, J. (2008). Failure of a new antivenom to treat Echis ocellatus snake bite in rural Ghana: the importance of quality surveillance. Transactions of the Royal Societyof Tropical Medicine and Hygiene. 102(5):445-450.

Wang, J., Al-Lamki, R.S., Zhang, H., Kirkiles-Smith, N., Gaeta, M.L., Thiru, S., (2003). Histamine antagonizes tumor necrosis factor (TNF) signalling by stimulating TNF receptor shedding from the cell surface and Golgi storage pool. Journal of Biological Chemistry. 278(24):21751-21760.

Warrell, D.A. (1995). Clinical toxicology of snakebite in Asia. In Handbook of Clinical Toxicology of Animal Venoms and Poisons. USA: Meier and White. Pp 493-594.

Warrell, D.A. (1999). Guidelines for the clinical management of snakebite in the Southeast Asian Region. South East Asian Journal of Tropical Medicine.and Public Health. 1:1-85.

World Health Organisation. (2010). Neglected tropical diseases: snakebite. Retrieved 12 October 2010. Accessed 5 April, 2015.

Zhou, X., Tan, T.C., Vallyaveettil, S., Go, M.L., Kini, R.M., VelazuezCampoy, A., Sivaraman, J. (2008). Structural characterization of myotoxic ecarpholin S from Echis carinatus venom. Biophysical Journal. 95:33663380 . 